\title{
Performance analysis of dual-branch selection combining technique over the generalized alpha-mu fading channels
}

\author{
Hasan Aldiabat ${ }^{1}$, Ahmed Alhubaishi ${ }^{2}$ \\ ${ }^{1}$ Department of Telecommunications Engineering, Yarmouk University, 21163 Irbid, Jordan \\ ${ }^{2}$ Department of Computer and Comm. Engineering, Universiti Putra Malaysia, Serdang 43400, Malaysia
}

\begin{abstract}
Article Info
Article history:

Received Jan 20, 2021

Revised Mar 22, 2021

Accepted Mar 30, 2021

\section{Keywords:}

Bit error rate

Diversity

Selection combining

$\alpha-\mu$ fading channels

ABSTRACT

Inspired by the low-difficulty of implementing a dual-branch selection combining (SC) technique, this research paper presents approximate closed form expressions for the bit error rate (BER) of M-ary phase shift keying (M-PSK) considering the SC technique. In particular, the BER expression is derived over independent and identically distributed (i.i.d) alpha - mu fading channels and is based on the use of Meijer's G-function. The presented mathematical formulas can be modified to study the performance of different types of fading channels including Weibull, exponential, Nakagami$\mathrm{m}$, Gamma, and Rayleigh channels. This can be achieved by updating the parameters of the propagation medium nonlinearity (alpha) and the number of multipath clusters $(m u)$. In addition, the paper provides numerical results that demonstrate a close match in the performance of the derived expressions and the simulation findings in terms of BER. Specifically, a very close to a total BER match is achieved using a range of signal to noise ratio (SNR) levels for various selections of the alpha and $m u$ parameters. The obtained closed form BER expression of M-PSK considering the dual-branch SC technique is novel, new, and has never been published in the literature before.
\end{abstract}

This is an open access article under the CC BY-SA license.

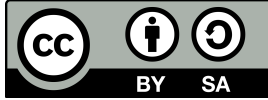

\section{Corresponding Author:}

Hasan Aldiabat

Department of Telecommunications Engineering

Yarmouk University

21163 Irbid, Jordan

Email: hasan.aldiabat@yu.edu.jo

\section{INTRODUCTION}

Propagation of radio waves through wireless communication channels is a sophisticated phenomenon which is heavily dependent on multipath fading [1]-[4]. As a result, most of the currently used wireless communication systems employ diversity to combat multipath fading effects. Diversity combining, the process of combining two or more replicas of the same information, is used to boost the reliability of wireless technologies by increasing the overall signal to noise ratio (SNR) at the receivers [5], [6].

The most common techniques of diversity combining are maximum ration combining (MRC), equal gain combining (EGC) and selection combining (SC) [7]-[10]. In MRC, both the amplitude and phase of each channel need to be understood at the receiver end in order to maximize the SNR at the combiner output. In EGC, all channels are equally weighted and only the channel phases need to be understood at the receiver end. However, for SC no need to estimate both the channel amplitudes and the channel phases. This is due to the fact that SC selects the channel branch of the highest SNR level. Therefore, SC is the least complex diversity combining technique to implement relative to MRC and EGC [11]-[15]. 
The multipath fading in wireless communications can be modeled by the [16]-[17]. It is a broad, adaptable and mathematically attractable distribution that explores the nonlinearity and clustering phenomena of wireless radio propagation media. The alpha parameter is related to the propagation medium nonlinearity, whereas the $m u$ parameter is related to the number of multipath clusters. Several distribution models, including Gamma, Weibull, Nakagami-m, exponential, and Rayleigh models, can easily be obtained from the alpha-mu fading distribution. It is possible to do this by substituting certain values for the alpha and mu parameters.

Many researchers have investigated the alpha-mu fading distribution's characteristics. The author of [16] introduced the level-crossing frequency, the length of average fading, and the joint statistics of the correlated alpha-mu variables. The statistical moments of the alpha-mu fading distribution were derived in [18]. In addition, reference [18] evaluates the average error rate of coherent modulation techniques using the moment generating function approach over this generalized fading distribution. In [19], the researchers came up with mathematical formulas for the degree of fading and the average normalized channel capacity of the distribution. The authors of [20] characterized the asymptotic error probability at large levels of SNR in reference to the fading parameters alpha and $m u$. Moreover, they expanded their research to include multipleinput multiple-output (MIMO) wireless communication systems that use orthogonal space time block coding (OSTBC). In [2], infinite series mathematical formulas were provided for the probability distribution (PDF) and cumulative distribution (CDF) functions of the signal to interference ratio (SIR) over correlated alpha$m u$ fading channels with dual SC diversity receiver. This research article provides a new formulation for the bit error rate (BER) performance of M-PSK systems over alpha-mu fading channels using dual branch SC technique. The formulation is based on the use of Meijer's G-function to solve complex integrals.

The remainder of this research paper is set out as follows: a description for the alpha-mu fading distribution and the SC system model is introduced in Section 2. Section 3 provides the BER analysis of M-PSK systems utilizing dual-branch SC. Numerical findings and simulation results are given in Section 4. Finally, in Section 5, the conclusions have been drawn.

\section{SYSTEM AND CHANNEL MODELS}

In this research article, a wireless communication system that transmits data over independent and identically distributed (i.i.d) fading channels is assumed. In particular, we consider the fading channel envelope, $R$, that follows the alpha-mu fading distribution and has a PDF expression defined as [16]:

$$
f_{R}(r)=\frac{\alpha \mu^{\mu} r^{\alpha \mu-1}}{\Gamma(\mu) \times \tilde{r}^{\alpha \mu}} \times e^{-\mu\left(\frac{r}{\tilde{r}}\right)^{\alpha}}, \quad \alpha>0, \quad \mu>0
$$

Where alpha is the nonlinearity and fading parameter, and $\mu$ is related to the normalized variance as the following:

$$
\mu=\frac{E^{2}\left[R^{\alpha}\right]}{V\left[R^{\alpha}\right]}
$$

Where $V[\cdot]$ and $E[\cdot]$ are the operators for the variance and expectation, respectively. The parameters $\Gamma(\cdot)$ and $\tilde{r}$ are the Gamma function $\left(\Gamma(x)=\int_{0}^{\infty} y^{x-1} \times e^{-y} d y\right)$ and the $\alpha$-root mean value of the envelope $\left(\tilde{r}=\left(E\left[R^{\alpha}\right]\right)^{\frac{1}{\alpha}}\right)$, respectively. We assume the transmitter can send data traffic via two i.i.d alpha-mu fading channels and the receiver employs SC diversity scheme. It is known that at the receiver side with SC diversity scheme, the receiver selects the branch of the largest instantaneous level of SNR, $\gamma$, where $\gamma=\max \left(\gamma_{1}, \gamma_{2}\right)$ with $\gamma_{i}=h_{i}^{2} \frac{E_{s}}{N_{o}}$ is the instantaneous SNR of the $i^{t h}$ path. The $E_{s}, h_{i}$, and $N_{o}$ represent the symbol energy, the instantaneous gain of the $i^{t h}$ path, and the noise power spectral density, respectively. The probability density of $\gamma_{i}$ is defined as [16]:

$$
f_{\gamma i}(\gamma i)=\frac{\alpha \mu^{\mu} \times \gamma_{i}^{\frac{\alpha \mu}{2}-1}}{2 \Gamma(\mu) \times{\widetilde{\gamma_{i}}}^{\frac{\alpha \mu}{2}}} \times e^{-\mu\left(\frac{\gamma_{i}}{\gamma_{i}}\right)^{\alpha / 2}}
$$

where $\tilde{\gamma}_{i}=E\left[\tilde{r}_{i}^{2}\right] \frac{E_{s}}{N_{o}}$. 


\section{AVERAGE BER WITH DUAL-BRANCH SC}

Herein, we address two main aspects of this article. we provide derivations for the PDF of the alpha$m u$ distribution considering SC via $i . i . d$ channels. In addition, we define the average BER of M-PSK modulation scheme via $i . i . d \alpha-\mu$ fading channels considering dual branch SC as a combining technique at the receiver side.

\subsection{PDF of alpha-mu distribution with SC}

It is known that the PDF of any distribution can be found by deriving its CDF. The alpha-mu fading distribution's CDF is written as [16]-[18]:

$$
F(\gamma)=\Gamma^{-1}(\mu) \times \gamma\left(\mu, \mu\left(\frac{\gamma}{\tilde{\gamma}}\right)^{0.5 \alpha}\right)
$$

Where $\gamma(x, y)$ in the numerator represents the incomplete gamma function and is given by $\gamma(x, y)=$ $\int_{0}^{x} z^{x-1} e^{-z} d z$. The PDF of $L$ branches SC diversity combining scheme is obtained by differentiating the CDF of the fading channels raised to power $L$. By following the procedure used in [22], the combined SNR PDF at the output of the dual-branch SC combiner is given by [23].

$$
f_{\gamma}^{S C}(\gamma)=\gamma^{\frac{\alpha \mu}{2}-1} e^{-\mu\left(\frac{\gamma}{\tilde{\gamma}}\right)^{0.5 \alpha}} \times \frac{\alpha \mu^{\mu}}{\Gamma(\mu) \tilde{\gamma}^{\frac{\alpha \mu}{2}}} \times \frac{\gamma\left(\mu, \mu\left(\frac{\gamma}{\tilde{\gamma}}\right)^{\alpha / 2}\right)}{\Gamma(\mu)}
$$

After representing the definition of $\gamma(x, y)$ by its equivalent finite series expression that is given by [23].

$$
\gamma(j, k)=(j-1) !\left(1-e^{-k} \sum_{n=0}^{j-1} \frac{k^{n}}{n !}\right),
$$

we can write the combined SNR PDF located at the dual-branch SC output as:

$$
f_{\gamma}^{S C}(\gamma)=\frac{\alpha \mu^{\mu} \times(\mu-1) !}{(\Gamma(\mu))^{2} \times \tilde{\gamma}^{\frac{\alpha \mu}{2}}} \times \gamma^{\frac{\alpha \mu}{2}-1} \times e^{-\mu\left(\frac{\gamma}{\bar{\gamma}}\right)^{0.5 \alpha}} \times\left(1-e^{-\mu\left(\frac{\gamma}{\bar{\gamma}}\right)^{0.5 \alpha}} \sum_{n=0}^{\mu-1} \frac{\gamma^{\frac{\alpha n}{2}}\left(\frac{\mu}{\tilde{\gamma}^{0.5 \alpha}}\right)^{n}}{n !}\right)
$$

which is a suitable form to be used for the purpose of finding the BER of communication schemes that employ coherent modulations.

\subsection{Average BER of M-PSK}

In [24], bit error rate approximations of coherent M-PSK modulation scheme are provided assuming Gray code bit mapping [25]. The approximations are reported in [26] and considered accurate expressions because they are valid for high as well as low SNR levels. The final expression of BER for the coherent M-PSK modulation scheme is written as:

$$
P_{b} \approx \frac{2}{\max \left(\log _{2}(M), 2\right)} \times \sum_{j=1}^{\max \left(\frac{M}{4}, 1\right)} Q\left(\sin \left((2 j-1) \frac{\pi}{M}\right) \times \sqrt{2 \gamma \log _{2}(M)}\right)
$$

It is possible to determine the average BER of M-PSK modulation scheme by averaging the error rate given in (8) over the PDF of the alpha-mu fading channel with SC given in (7) as:

$$
P_{b}, a v g=\int_{0}^{\infty} P_{b} \times f_{\gamma}^{S C}(\gamma) d \gamma
$$

After substituting as shown in (7) and (8) into (9), we get 


$$
\begin{aligned}
P_{b}, \operatorname{avg}= & \frac{2}{\max \left(\log _{2}(M), 2\right)} \times \frac{\alpha \mu^{\mu}(\mu-1) !}{\Gamma(\mu)^{2} \times \widetilde{\gamma}^{\frac{\alpha \mu}{2}} \times} \\
& \sum_{j=1}^{\max \left(\frac{M}{4}, 1\right)} \int_{0}^{\infty}\left\{Q\left(\sin \left((2 j-1) \frac{\pi}{M}\right) \times \sqrt{2 \gamma \log _{2}(M)}\right) \times\right. \\
& \left.\gamma^{\frac{\alpha \mu}{2}-1} \times e^{-\mu\left(\frac{\gamma}{\bar{\gamma}}\right)^{0.5 \alpha}}\left(1-e^{-\mu\left(\frac{\gamma}{\tilde{\gamma}}\right)^{0.5 \alpha}} \times \sum_{n=0}^{\mu-1} \frac{\left(\frac{\mu}{\tilde{\gamma}^{0.5 \alpha}}\right)^{n}}{n !} \times \gamma^{\frac{\alpha n}{2}}\right)\right\} d \gamma
\end{aligned}
$$

By using the definitions of the Q function and the Meijer's G function [27], the integral as shown in (10) can be expressed by the following:

$$
\begin{aligned}
I=\frac{\Gamma(1 / 2) k^{1 / 2}}{l \times(2 \pi)^{\frac{k+l}{2}}} & {\left[\left(\frac{l}{\log _{2}(M) \times\left(\sin \left((2 j-1) \frac{\pi}{M}\right)\right)^{2}}\right)^{\frac{\alpha \mu}{2}} \times\right.} \\
& G_{2 l, k+l}^{k, 2 l}\left(\begin{array}{c}
\Delta\left(l, \frac{1-\alpha \mu}{2}\right), \Delta\left(l, 1-\frac{\alpha \mu}{2}\right) \\
\Delta(k, 0), \Delta\left(l,-\frac{\alpha \mu}{2}\right)
\end{array} \mid \frac{x}{\left(\sin \left((2 j-1) \frac{\pi}{M}\right)\right)^{2 l}}\right) \\
& -\sum_{m=0}^{\mu-1} \frac{\left(\frac{\mu}{\tilde{\gamma}^{\alpha / 2}}\right)^{m}}{m !}\left(\frac{1}{m^{2}}\right) \\
& \left.G_{2 l, k+l}^{k, 2 l}\left(\begin{array}{c}
\Delta\left(l, \frac{1-\alpha \mu}{2}\right), \Delta\left(l, 1-\frac{\alpha \mu}{2}\right) \\
\Delta(k, 0), \Delta\left(l,-\frac{\alpha \mu}{2}\right)
\end{array} \mid \frac{x}{\left(\sin \left((2 j-1) \frac{\pi}{M}\right)\right)^{2 l}}\right)\right]
\end{aligned}
$$

Where $l$ and $k$ represent integer numbers chosen based on the criteria that $l / k=\alpha / 2$ with a great common divisor of one. The $x$ and $\Delta(\cdot)$ are defined as:

$$
x=\frac{\mu^{k} \times l^{l}}{\tilde{\gamma}^{\alpha k / 2}\left(\log _{2} M\right)^{l} \times k^{k}} \text { and } \Delta(\nu, \kappa)=\nu / \kappa,(\nu+1) / \kappa, . .,(\nu+\kappa-1) / \kappa \text {. It is worth mentioning that }
$$

Meijer's G-function $\left(G_{p, q}^{m, n}\right)$ is readily available as pre-installed feature in several mathematical software tools including MATLAB, MATHEMATICA and MAPLE. Upon substituting $I$ into as shown in (10), we can write the average BER as shown in (12).

$$
P_{b, a v e} \approx \frac{2}{\max \left(\log _{2} M, 2\right)} \times C \times \sum_{j=1}^{\max \left(\frac{M}{4}, 1\right)} I,
$$

where $C=\frac{\alpha \times \mu^{\mu} \times(\mu-1) !}{(\Gamma(\mu))^{2} \times \tilde{\gamma}^{\alpha \mu / 2}}$ and $I$ as given in (11).

The expression given in (12) represents the final result of the average BER of wireless communication systems that use M-PSK over i.i.d alpha-mu fading channels and utilize dual branch SC diversity combining scheme.

\section{ANALYSIS AND NUMERICAL RESULTS}

This section of the paper demonstrates numerically the benefits of exploiting dual branch SC diversity technique over wireless communication channels. In addition, it presents results that show the agreement between the derived analytical expressions in the paper and simulation findings.

Employing dual-branch SC with wireless communication systems can reduce the average bit error rate (BER) effectively. Figure 1 demonstrates the average BER results with (red curves) and without (black curves) using dual-branch SC over Rayleigh fading channels, a particular instance of the alpha - mu channel when $a l p h a=2$ and $m u=1$. The results are generated for a communication system that uses two different PSK constellation sizes. The results demonstrated in Figure 1 (a) are for 4PSK and the ones in Figure 1 (b) 
are for 16 PSK. It is clear from the figures that using dual branch SC (red curves) reduces the average BER of the communication system for both low and high SNR levels. In addition, the figures demonstrate that the improvement is more significant for high SNR levels. Even though the presented results are for 4PSK and 16PSK constellation sizes, but they are the trend for any M-PSK constellation size.

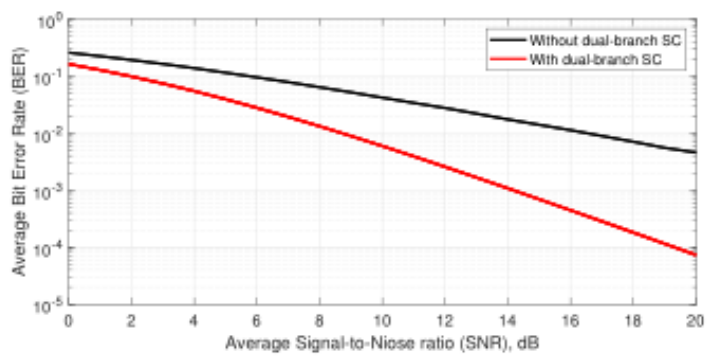

(a)

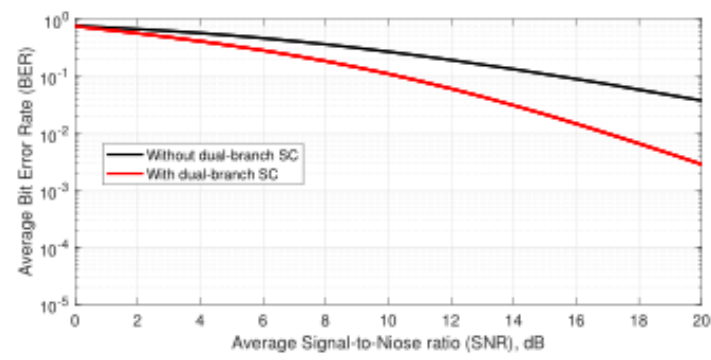

(b)

Figure 1. Average BER for $\alpha=2, \mu=1$ with and without using dual-branch SC using; (a) 4PSK; (b) 16PSK modulation schemes

The agreement between the derived analytical expressions and simulation findings is demosntarated for a particular set of alpha and $m u$ parameters as well as for a variety of M-PSK constellation sizes. Figure 2 displays the behavior of average BER versus average SNR using alpha $=2$ and $m u=1$, in other words, Rayleigh fading channels. Specifically, Figure 2 (a) demonstrates the analytical and simulation results when 4PSK and 16PSK modulation schemes are used, whereas Figure 2 (b) demonstrates the results for 32PSK and 64PSK modulation schemes. The two figures show a close to a total match between the simulation and analytical results. For example, for 4PSK and 16PSK in Figure 2 (a) as well as for 64PSK in Figure 2 (b) the results are almost in a total match, and they are close for the case of 32PSK as demonstrated in Figure 2 (b). The numerical findings of average BER versus the average SNR over Nakagami-2, alpha $=2$ and $m u=2$, fading channels are provided in Figure 3. Figure 3 (a) demonstrates the findings when 4PSK and 16PSK are used and Figure 3 (b) shows the findings for 32PSK and 64PSK. From the figures, one can clearly see the match between the simulation and analytical results specially for high SNR values.

Figures 4 (a) and (b) are generated under the same conditions, i.e. dual-branch SC via i.i.d alpha-mu fading channels, however considering the Weibull fading distribution $(\alpha=3, \mu=1)$. Figure 4 (a) shows the analytical and simulation results when using 4PSK and 16PSK, and Figure 4 (b) shows the results when using 32PSK and 64PSK modulation schemes. Again, the simulation results exhibited a close to a total agreement with the analytical ones. The same situations are considered for Nakagami-3, alpha $=2$ and $m u=3$, fading distribution as demonstrated in Figure 5.

Repeatedly, a close match among the analytical and simulation findings of the average BER over the used modulation schemes is shown. The 4PSK and 16PSK modulation schemes are considered in Figure 5 (a), and the 32PSK and 64PSK modulation schemes are considered in Figure 5 (b). In all the presented results, we used MATLAB software to evaluate Meijer's function presented in Section 3 and to plot the curves of BER values.The only limitation of using SC diversity technique is that it has a low output SNR for a given number of diversity branches as compared to other combining diversity techniques, such as MRC and EGC. However, SC diversity technique does not require coherent reception and it has a low complexity as compared to the other combining techniques. This is due to the fact that SC exploits only one of the used diversity branches, therefore, no need to estimate the channel fading phases and amplitudes of each diversity branch. 


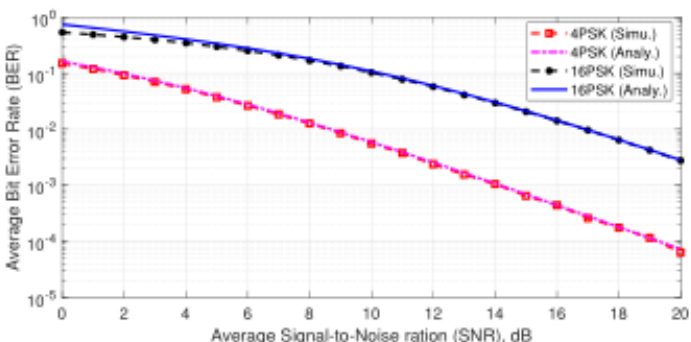

(a)

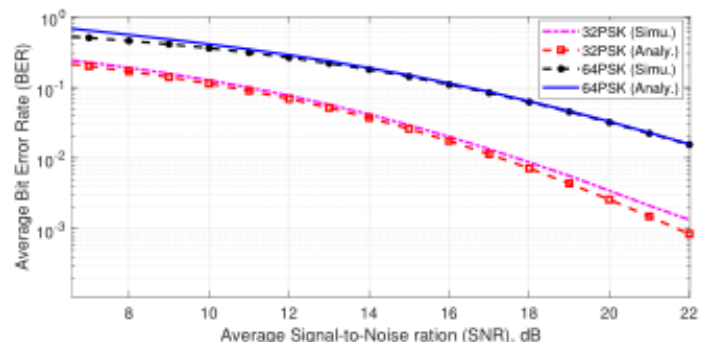

(b)

Figure 2. Average BER for $\alpha=2, \mu=1$ (Rayleigh fading channels) using: (a) 4PSK and 16PSK, (b) 32PSK and 64PSK modulation schemes

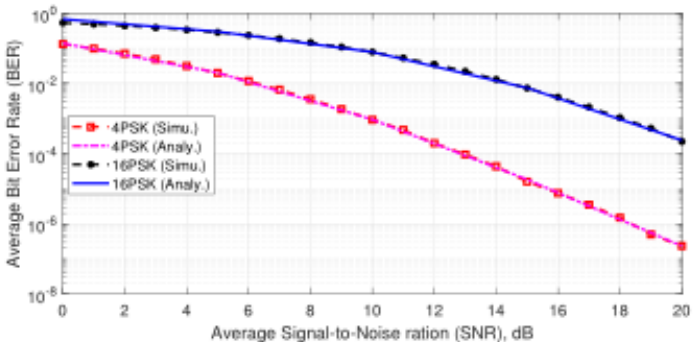

(a)

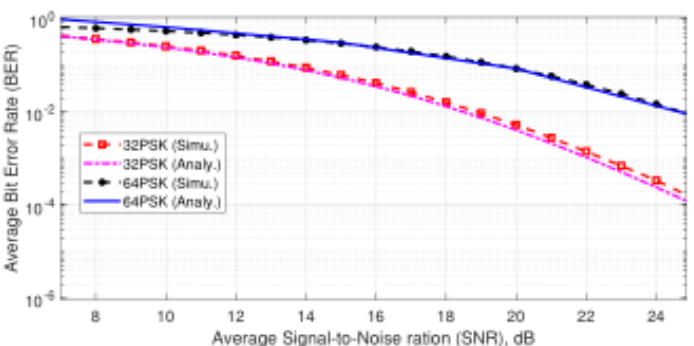

(b)

Figure 3. Average BER for $\alpha=2, \mu=2$ (Nakagami-2 fading channels) using; (a) 4PSK and 16PSK; (b) 32PSK and 64PSK modulation schemes

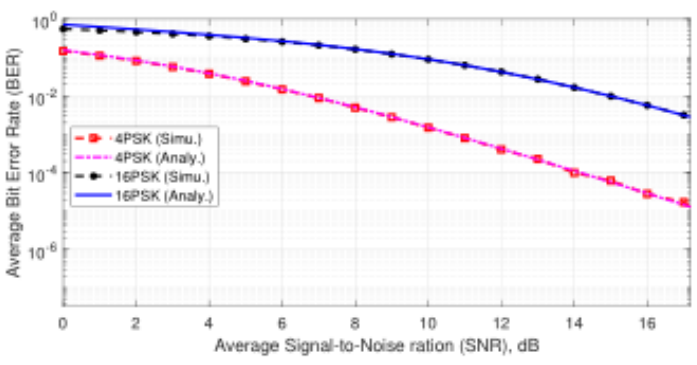

(a)

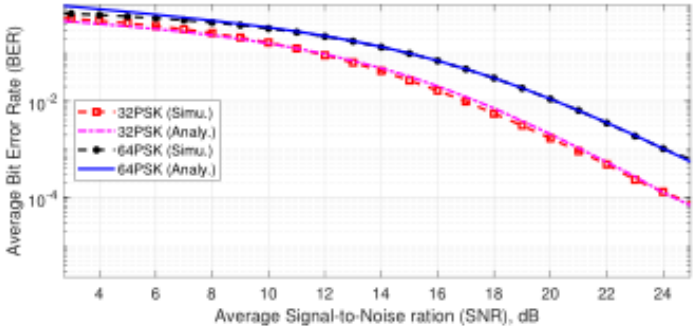

(b)

Figure 4. Average BER for $\alpha=3, \mu=13$ (Weibull fading channels) using; (a) 4PSK and 16PSK; (b) 32PSK and 64PSK modulation schemes

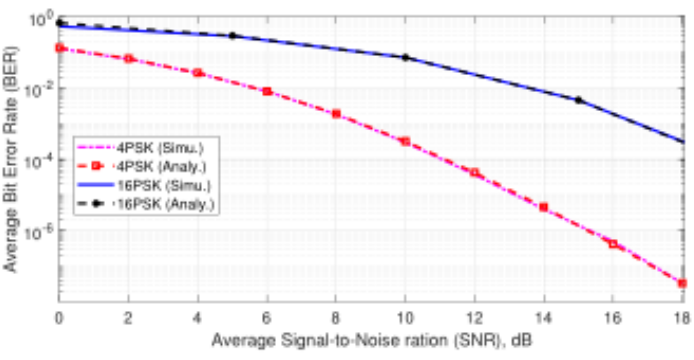

(a)

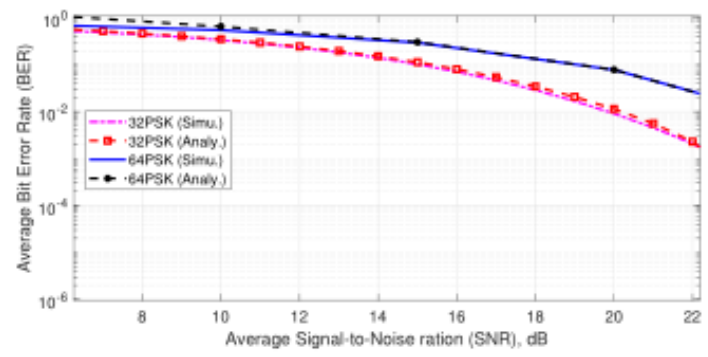

(b)

Figure 5. Average BER for $\alpha=2, \mu=3$ (Nakagami-3 fading channels) using; (a) 4PSK and 16PSK; (b) 32PSK and 64PSK modulation schemes 


\section{CONCLUSIONS}

This paper presented a closed mathematical formula for the average bit error rate of M-PSK modulation scheme under the impact of dual branch SC via independent and identically distributed alpha-mu fading channels. The formula can be reduced to include other fading distributions, including Rayleigh, Weibull, exponential, Nakagami-m, one-sided Gaussian, and Gamma distributions, by selecting appropriate values for the alpha and $m u$ variables. The numerical results exhibited a close to a total match between the analytical and simulated outputs. The work presented in this paper can be extended to consider correlated alpha - mu channels, where correlation coefficient between the faded channels will have an effect on the combined SNR PDF at the output of the diversity system.

\section{REFERENCES}

[1] Badarneh, Osamah S., Daniel Benevides da Costa, Mustapha Benjillali, and Mohamed-Slim Alouini, "Selection Combining Over Double $\alpha-\mu$ Fading Channels," IEEE Transactions on Vehicular Technology, vol. 69, no. 3, pp. 3444-3448, Mar. 2020, doi: 10.1109/TVT.2020.2969224.

[2] S. Panic, M. C. Stefanovic, and A. V. Mosic, "Performance analyses of selection combining diversity receiver over [alpha]-[mu] fading channels in the presence of co-channel interference," IET communications, vol. 3, no. 3, pp. 1769 - 1777, Dec. 2009, doi: 10.1049/iet-com.2009.0023.

[3] S. Prakash, I. McLoughlin, "Effects of channel prediction for transmit antenna selection with maximalratio combining in rayleigh fading," IEEE transactions on vehicular technology, vol. 60, no. 6, pp. 25552568, Jul. 2011, doi: 10.1109/TVT.2011.2157184.

[4] Awon, Nuzhat Tasneem, Md Islam, Md Rahman, and A. Z. M. Islam, "Effect of AWGN \& Fading (Raleigh \& Rician) channels on BER performance of a WiMAX communication System," arXiv preprint, vol. 10, no. 8, Nov. 2012.

[5] Darawsheh, Hikmat Y., and Ali Jamoos, "Selection diversity combining analysis of energy detector over $\alpha-\mu$ generalized fading channels," The International Conference on Technological Advances in Electrical, Electronics and Computer Engineering (TAEECE), May 2013, doi: 10.1109/TAEECE.2013.6557336.

[6] Ikki, Salama, and Mohamed H. Ahmed, "Performance analysis of cooperative diversity wireless networks over Nakagami-m fading channel,' IEEE Communications letters, vol. 11, no. 4, pp. 334-336, Apr. 2007, doi: 10.1109/LCOM.2007.348292.

[7] Varzakas, Panagiotis. "Optimal SIR for an hybrid cellular DS/SFH CDMA system," International Journal of Communication Systems 21, no. 5, pp. 549-557, May 2008, doi: 10.1002/dac.897.

[8] Brennan, Donald G, "Linear diversity combining techniques," Proceedings of the IRE, vol. 47, no. 6, pp. 1075-1102, Feb. 2003, doi: 10.1109/JPROC.2002.808163.

[9] Stber, Gordon L, ’Principles of mobile communication,” Springer Publishing Company, 2011.

[10] Rappaport, Theodore S., "Wireless communications: principles and practice," vol. 2. New Jersey: prentice hall PTR, 1996.

[11] Le, Khoa N.,"A review of selection combining receivers over correlated Rician fading," Digital Signal Processing, vol. 88, pp. 1-22, May 2019, doi: 10.1016/j.dsp.2019.01.015.

[12] Khatalin, Sari, "Performance of dual-branch selection combining diversity systems in non-identical Nakagami-q (Hoyt) fading channels," IET communications, vol. 4, no. 5, pp. 585-595, Apr. 2010, doi: 10.1049/iet-com.2009.0445.

[13] Golubovic, Aleksandra D., Nikola M. Sekulovic, Mihajlo C. Stefanovic, Dejan N. Milic, and Zorica B. Nikolic, "Performance Analysis of a Selection Combining System That Uses the Minimal Interference Algorithm," IETE Journal of Research, pp. 1-7, Apr. 2019, doi: 10.1080/03772063.2019.1604174.

[14] Lema, G. G., T. B. Reda, D. Hailu, and T. Sutikno, "Performance evaluation of space time trellis coded MIMO for mobile communications," Indonesian Journal of Electrical Engineering and Computer Science, vol. 15, no. 3, pp. 1501-1508, Sept. 2019, doi: 10.11591/ijeecs.v15.i3.pp1501-1508.

[15] Phan, Van-Duc, Tan N. Nguyen, Minh Tran, and Thanh-Long Nguyen, "Outage probability analysis of dual energy harvesting relay network over rayleigh fading channel using SC and MRC technique," Indonesian Journal of Electrical Engineering and Computer Science, vol. 16, no. 2, pp. 803-811, Nov. 2019, doi: 10.11591/ijeecs.v16.i2.pp803-811.

[16] Yacoub, Michel Daoud, "The $\alpha-\mu$ Distribution: A Physical Fading Model for the Stacy Distribution," IEEE Transactions on Vehicular Technology, vol. 56, no. 1, pp. 27-34, 2007. 
[17] Lei, Hongjiang, Imran Shafique Ansari, Gaofeng Pan, Basel Alomair, and Mohamed-Slim Alouini, "Secrecy capacity analysis over $\alpha-\mu$ fading channels," IEEE Communications Letters, vol. 21, no. 6, pp. 1445-1448, 2017.

[18] Magableh, Amer M., and Mustafa M. Matalgah, "Moment generating function of the generalized $\alpha-\mu$ distribution with applications," IEEE Communications Letters, vol. 13, no. 6, pp. 411-413, Jun. 2009, doi: 10.1109/LCOMM.2009.090339.

[19] Magableh, Amer M., and Mustafa M. Matalgah, "Channel characteristics of the generalized alpha-mu multipath fading model," In 2011 7th International Wireless Communications and Mobile Computing Conference, Jul. 2011, doi: 10.1109/IWCMC.2011.5982766.

[20] Song, Youngpil, Hyundong Shin, and Wonha Kim, "Asymptotic SEP for M-PSK Signals over $\alpha$ - $\mu$ Fading Channels." IEEE communications letters, vol. 12, no. 9, pp. 675-677, 2008.

[21] Alouini, M-S., and Andrea J. Goldsmith, "Capacity of Rayleigh fading channels under different adaptive transmission and diversity-combining techniques," IEEE transactions on Vehicular Technology, vol. 48, no. 4, pp. 1165-1181, Jul. 1999, doi: 10.1109/25.775366.

[22] Aldalgamouni, Taimour, Amer M. Magableh, and Ahmad Al-Hubaishi, "Performance of Selected Diversity Techniques Over The $\alpha-\mu$ Fading Channels", WSEAS Transaction communications, 2013.

[23] Gradshteyn, Izrail Solomonovich, and Iosif Moiseevich Ryzhik, "Table of integrals, series, and products," Academic press, 2014.

[24] Lu, Jianhua, Khaled Ben Letaief, JC-I. Chuang, and Ming L. Liou, "M-PSK and M-QAM BER computation using signal-space concepts," IEEE Transactions on communications, vol. 47, no. 2, pp. 181-184, Feb. 1999, doi: 10.1109/26.752121.

[25] Lee, Pil, "Computation of the bit error rate of coherent M-ary PSK with Gray code bit mapping," IEEE Transactions on Communications, vol. 34, no. 5, pp. 488-491, May 1986, doi: 10.1109/TCOM.1986.1096558.

[26] Simon, Marvin K., and Mohamed-Slim Alouini, ”Digital communication over fading channels," John Wiley \& Sons, 2005.

[27] Prudnikov, Anatoliı Platonovich, Yu A. Brychkov, Oleg Igorevich Marichev, and Robert H. Romer,'Integrals and series," CRC Press, 1988.

\section{BIOGRAPHIES OF AUTHORS}

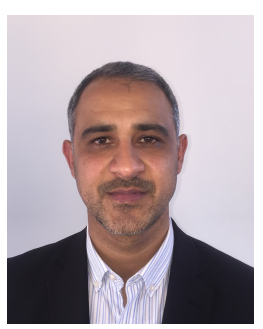

Hasan Aldiabat received his B.Sc. and M.Sc. degrees in Electrical Engineering form Jordan University of Science and Technology, Irbid, Jordan, in 2009 and 2011, respectively. He received his $\mathrm{Ph} . \mathrm{D}$. degree in Electrical and Computer Engineering from the University of Minnesota, Minneapolis, USA, in 2019. He is currently Assistant Professor with the Department of Telecommunications Engineering at Yarmouk University, Irbid, Jordan. His research interest is in fields of ultrasound arrays, signal processing, and wireless communications.

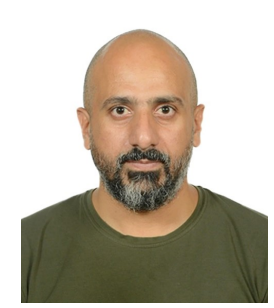

Ahmed Alhubaishi received his B.Sc. in Telecommunications Engineering form Mutah University, Karak, Jordan, in 2008. He is currently a Ph.D. candidate in Communications Engineering at Universiti Putra Malaysia, Serdang 43400, Malaysia. His research interest is in fields of signal processing, networks, and wireless communications. 